\title{
EXPONENTIAL DECAY FOR A THERMOELASTIC BEAM BETWEEN TWO STOPS
}

\section{Santina Arantes and Jaime Rivera}

National Laboratory for Scientific Computation - LNCC, Petrópolis, Rio de Janeiro, Brazil

A one-dimensional initial-boundary value problem for a thermoelastic beam that is clamped at one end and moves freely between two obstacles at the other, is studied using a method of functional analysis. The boundary conditions at the free end are represented either by classic Signorini's conditions or a normal compliance condition. The main result is that the energy associated with the system decays exponentially as time goes to infinity. Numerical examples are included.

Keywords: Contact problem; Dynamic vibrations; Normal compliance condition; Penalized problem; Signorini's conditions; Thermoelastic beam.

\section{INTRODUCTION}

The study of dynamic vibrations of mechanical systems caused, for example, by noises and characteristic vibrations of cars and its components became an area of increasing interest for the automotive industries. These industries have employed great efforts in the sense to identify and to reduce these unwanted noises and vibrations. The dynamic vibrations problem of beams in contact with a rigid obstacle has been studied for some authors, such as: Kuttler et al. [1], Andrews et al. [2], Dumont et al. [3] and Bajkowski et al. [4]. The one-dimensional problem describing the viscoelastic or elastic oscillations of a beam clamped at one end to a vibrations device while the other end oscillates between two rigid or flexible stops, was first studied by Moon and Shaw in [5]. This model was also studied by Kuttler and Shillor in [6], where the authors consider the material constitutive law to be either elastic or viscoelastic of the Kelvin-Voigt type. The contact is modeled either with the Signorini's conditions or with the normal compliance condition. The authors show the existence of weak solutions and the uniqueness is proved only for the viscoelastic case. In reference [7], Dumont and Paoli study this problem considering the elastic case and analyse the convergence of a full discretized approximation; prove the stability and convergence of model and present implementation results. For other numerical results see, for example, $[4,8]$ and references therein.

Received 11 April 2007; accepted 28 January 2008.

Address correspondence to Santina de Fatima Arantes, Laboratorio Nacional de Computacao Cientifica, Rua Getulio Vargas, 333 Quitandinaha, Petropolis-Rio de Janeiro 25651-070, Brazil. E-mail: santina@Incc.br 
It is well known that beams that vibrate with high frequency may generate considerable heat. For this reason, in this work we take into account the heat generated during the process. The model includes dynamic vibrations of the beam and the associated generation of heat; that is, the thermoelastic beam problem. Because of the lack of uniqueness for the Signorini's problem, the main contribution of this work is to show that there exists one solution of the Signorini's problem, which decays exponentially to zero as time goes to infinity. We also present numerical simulations that verify our theoretical results.

Throughout this article the same letter $C_{0}$ will denote various positive constants that do not depend on $x$ and $t$, and $C_{\delta}=C_{0} \delta^{-1}$ where $\delta$ is any positive constant.

\section{MODEL FORMULATION}

We consider a thermoelastic beam problem. The physical description of the model is given in Figure 1. The beam is clamped at its left end and its right end is free. The motion in the free end is constrained by two obstacles that are called stops.

We assume that the beam is configured in $[0, L] \subset \mathbb{R}$. Here $g_{1}$ and $g_{2}$, with $g_{1}<0<g_{2}$, are the positions of the stops. We make the usual Euler-Bernoulli assumption that the transverse dimensions of the beam are small enough compared to the length of the beam and radius of curvature of the bending that any plane cross-section, initially perpendicular to the axis of the beam, remains plane and perpendicular to the neutral surface during bending. The neutral surface is the one running through the length of the beam that suffers no extension or contraction during its bending.

If the beam has a uniform square cross-section with vertical thickness $h$, then the thermal moment at $(\tilde{x}, \tilde{t})$ is given by

$$
\tilde{\theta}=\tilde{\theta}(\tilde{x}, \tilde{t})=\frac{12}{h^{3}} \int_{-h / 2}^{h / 2} \tilde{y} \tilde{\Theta}(\tilde{x}, \tilde{y}, \tilde{t}) d \tilde{y}
$$

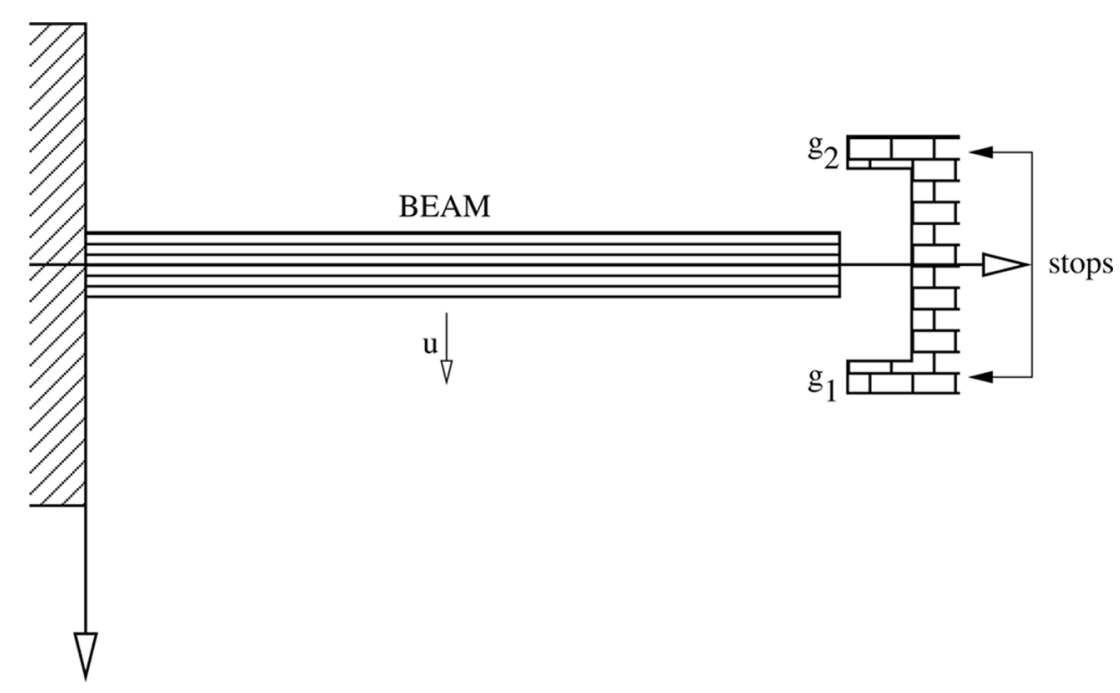

Figure 1 Beam subject to a constraint at the free end $L$. 
where $\widetilde{\Theta}(\tilde{x}, \tilde{y}, \tilde{t})$ is the temperature (measured relative to that of the environment) at $(\tilde{x}, \tilde{t})$ and vertical location $\tilde{y}$. In the discussion of the model that follows, we use the terms temperature difference and thermal moment interchangeably. We also denote by $\tilde{u}=\tilde{u}(\tilde{x}, \tilde{t})$ the vertical displacement and the subscripts the partial derivatives. The equations that describe thermoelastic beams follow from [9], where the author derived the equations [10]. Thus, the complete model is given by

$$
\begin{gathered}
\rho h \tilde{u}_{\tilde{t} \tilde{t}}+D \tilde{u}_{\tilde{x} \tilde{x} \tilde{x} \tilde{x}}+D \alpha(1+v) \tilde{\theta}_{\tilde{x} \tilde{x}}=\tilde{f} \text { in }(0, L) \times(0, \infty) \\
C \tilde{\theta}_{\tilde{t}}-k \tilde{\theta}_{\tilde{x} \tilde{x}}-\frac{\alpha T_{0} E}{1-v} \tilde{u}_{\tilde{x} \tilde{x} \tilde{x}}=\tilde{g} \text { in }(0, L) \times(0, \infty)
\end{gathered}
$$

the constants which appear in the above equations are given in Table 1.

We introduce the following change of variables to set the problem in a dimensionless form:

$$
x=\frac{\tilde{x}}{L}, \quad t=\frac{k \tilde{t}}{C L^{2}}, \quad \theta(x, t)=\frac{\tilde{\theta}(x, t)-T_{0}}{T_{0}}, \quad u(x, t)=\frac{\tilde{u}}{L} \frac{D}{C T_{0} L^{4}}
$$

This change is to replace the interval $[0, L]$ by the unit interval $[0,1]$ and to replace the temperature by $\theta=0$.

Considering all dimensionless variables, the shearing force per unit area of the beam $\sigma=\sigma(x, t)$ is given by the relation

$$
\sigma(x, t)=-u_{x x x}-b \theta_{x}
$$

and the heat flux is given by

$$
q(x, t)=-\theta_{x}
$$

So that, the complete model, in dimensionless variables, is given by

$$
\begin{gathered}
a u_{t t}+u_{x x x x}+b \theta_{x x}=f \quad \text { in }(0,1) \times(0, \infty), \\
\theta_{t}-\theta_{x x}-c u_{x x t}=g \text { in }(0,1) \times(0, \infty),
\end{gathered}
$$

where $a, b$ and $c$ are dimensionless positive constants given by

$$
a=\frac{\rho h k^{2}}{C^{2} D}, \quad b=\frac{D \alpha(1+v)}{L^{3} C}, \quad c=\frac{\alpha T_{0} E L^{3}}{(1-v) D}
$$

We consider the initial conditions

$$
u(x, 0)=u_{0}(x), \quad u_{t}(x, 0)=u_{1}(x), \quad \theta(x, 0)=\theta_{0}(x), \quad 0 \leq x \leq 1,
$$

where $u_{0}, u_{1}$ and $\theta_{0}$ represent, respectively, the initial displacement, the velocity and temperature difference of the beam. We assume that the beam has been thermally insulated at the clamped end and kept at a zero temperature at. Under these conditions it is not difficult to show that the problem for Eqs. (4)-(5) is well posed. 
It would be natural to consider a general case where the temperature at the free end is time dependent. However, in the general case a proof of the exponential stability is not possible using our techniques. Thus, for the sake of simplicity, we are taking the temperature null at this end (see $[4,11]$ ), so that the boundary conditions we consider for $\theta$ are

$$
\theta_{x}(0, t)=\theta(1, t)=0 \quad t>0
$$

We remark that we are assuming that there is not friction when the beam hits the obstacles, therefore there is not an extra generation of heat.

We assume that the beam is clamped at $x=0$, then

$$
u(0, t)=u_{x}(0, t)=0 \quad t>0
$$

and that the moment at the end $x=1$ is zero; that is

$$
u_{x x}(1, t)=0 \quad t>0
$$

At the free end of the beam, where contact with the obstacles may occur, we take the classic Signorini's condition

$$
g_{1} \leq u(1, t) \leq g_{2} \quad t>0
$$

This condition assures that the displacement at $x=1$ is constrained between the stops $g_{1}$ and $g_{2}$. When the beam hits one of the obstacles, the stress is in the opposite direction of the displacement. Then, we have the situations

$$
\begin{array}{ll}
\sigma(1, t)>0 & \text { if } u(1, t)=g_{1} \\
\sigma(1, t)<0 & \text { if } u(1, t)=g_{2} \\
\sigma(1, t)=0 & \text { if } g_{1}<u(1, t)<g_{2}
\end{array}
$$

To ensure that only one of the conditions occurs at the same time, we impose that

$$
\sigma(1, t)\left[g_{2}-u(1, t)\right]^{+}\left[u(1, t)-g_{1}\right]^{+}=0
$$

where $f^{+}=\max \{f, 0\}$ is the positive part of $f$.

To study this problem with Signorini's conditions, we consider first an approximation given by the normal compliance condition, where the stops are assumed to be flexible with resistance force proportional to the deflection. Introducing the penalization parameter $1 / \varepsilon$, we find the solution of the penalized problem, then we pass to the limit when $\varepsilon \rightarrow 0$ to find the solution of the Signorini's problem. The normal compliance condition is given by

$$
\sigma(1, t)=-k\left\{\left[u(1, t)-g_{2}\right]^{+}-\left[g_{1}-u(1, t)\right]^{+}\right\}
$$

where $k=1 / \varepsilon$ is a positive constant. This condition leads to a system of the partial differential equations depending on the parameter $\varepsilon$. Observe that the normal 
compliance condition (12) allows that the final contact penetrates the obstacle; that is, it may occurs that

$$
u(1, t)>g_{2} \text { or } u(1, t)<g_{1} \quad t>0
$$

From a mechanical point of view, $1 / \varepsilon$ can be interpreted as the stiffness of the stops. On the other hand, from numerical point of view, for large values of $1 / \varepsilon$, we obtain the rigid stops and the condition (11) can be seen as a limit case of the normal compliance condition (12) when $1 / \varepsilon$ tends to the infinity. Thus, the normal compliance condition (12) is called regularization of Signorini's conditions and says that condition (12) is an approximation of the problem with Signorini's conditions through the boundary conditions.

We used a beam of alloy steel, for which thermal and mechanics properties are given in Table 1.

Table 1 Material properties

\begin{tabular}{ll}
\hline Length & $L=1.2 \mathrm{~m}$ \\
\hline Thickness & $h=0.05 \mathrm{~m}$ \\
Density & $\rho=8000 \mathrm{~kg} / \mathrm{m}^{3}$ \\
Young's modulus & $E=207 \mathrm{GPa}$ \\
Poisson's ratio & $v=0.3$ \\
Coefficient of thermal expansion & $\alpha=11 \times 10^{-6} 1 /{ }^{\circ} \mathrm{C}$ \\
Modulus of flexural rigidity & $D=\frac{E h^{3}}{12\left(1-v^{2}\right)}=2.4 \times 10^{-3} \mathrm{GPam}$ \\
Heat capacity & $C=3.6 \times 10^{6} \mathrm{~kg} / \mathrm{m} \mathrm{s}{ }^{\circ} \mathrm{C}$ \\
Thermal conductivity & $k=38 \mathrm{~kg} \mathrm{~m} / \mathrm{s}^{3} \mathrm{C}$ \\
Reference temperature & $T_{0}=20^{\circ} \mathrm{C}$ \\
External sources & $\tilde{f}\left(\mathrm{~kg} / \mathrm{m} \mathrm{s}{ }^{2}\right)$ and $\tilde{g}\left(\mathrm{~kg} / \mathrm{m}^{2} \mathrm{~s}^{3}\right)$ \\
\hline
\end{tabular}

We take the values for $\rho$ from [7] and $E, v, \alpha, C$ and $k$ from [14]. The choice of $h$ have been taken small and the value for $T_{0}$ is the ambient temperature for the alloy steel.

\section{EXISTENCE OF SOLUTIONS AND VARIATIONAL FORMULATIONS}

Here we establish the existence of weak solutions as well as uniqueness for the penalized problem. We introduce the following spaces

$$
V=\left\{u \in H^{2}(0,1) ; u(0)=u_{x}(0)=0\right\}
$$

and

$$
E=\left\{\theta \in H^{1}(0,1) ; \theta(1)=0\right\}
$$

To incorporate the Signorini's conditions (10)-(11), we consider the convex set of all the admissible displacements in the right end of the beam.

$$
K=\left\{u \in V \quad g_{1} \leq u(1) \leq g_{2}\right\}
$$


We define $H=L^{2}(0,1)$ and we identify $H$ with $H^{\prime}$, topological dual of $H$, so we have

$$
V \subset H=H^{\prime} \subset V^{\prime} \text { and } E \subset H=H^{\prime} \subset E^{\prime}
$$

The classic formulation of the problem of vibrations of a thermoelastic beam between two obstacles with Signorini's conditions is: To find pairs of functions $\{u, \theta\}$, such that the system (4)-(11) is satisfied. The classical problem with normal compliance condition is: To find pairs of functions $\{u, \theta\}$ satisfying (4)-(9) and (12).

The definition of weak solution of the Signorini's problem (4)-(11), which we will use in this work is given as follows.

Definition 3.1. Assume that

$$
u_{0} \in K, \quad u_{1}, \theta_{0} \in L^{2}(0,1) \quad \text { and } f, g \in H^{1}\left(0, T ; L^{2}(0,1)\right)
$$

with $T$ any fixed positive real number. We say that the couple $\{u, \theta\}$ is a weak solution to (4)-(11), when

$$
\begin{aligned}
& u \in W^{1, \infty}\left(0, T ; L^{2}(0,1)\right) \cap L^{\infty}(0, T ; K) \\
& \theta \in L^{2}(0, T ; E) \cap L^{\infty}\left(0, T ; L^{2}(0,1)\right) \\
& u(x, 0)=u_{0}, \quad u_{t}(x, 0)=u_{1}, \quad \theta(x, 0)=\theta_{0} \\
& -a \int_{0}^{T} \int_{0}^{1} u_{t}\left(v_{t}-u_{t}\right) d x d t+\int_{0}^{T} \int_{0}^{1} u_{x x}\left(v_{x x}-u_{x x}\right) d x d t-b \int_{0}^{T} \int_{0}^{1} \theta_{x}\left(v_{x}-u_{x}\right) d x d t \\
& \quad \geq a \int_{0}^{1} u_{1}\left[v(x, 0)-u_{0}\right] d x+\int_{0}^{T} \int_{0}^{1} f(v-u) d x d t \\
& -\int_{0}^{T} \int_{0}^{1} \theta w_{t} d x d t+\int_{0}^{T} \int_{0}^{1} \theta_{x} w_{x} d x d t+c \int_{0}^{T} \int_{0}^{1} u_{x x} w_{t} d x d t \\
& \quad=\int_{0}^{1} \theta_{0} w(x, 0) d x-c \int_{0}^{1} u_{0 x x} w(x, 0) d x+\int_{0}^{T} \int_{0}^{1} g w d x d t \\
& \forall v \in L^{2}(0, T ; K), \quad \forall w \in L^{2}(0, T ; E), \quad \forall w_{t} \in L^{2}\left(0, T ; L^{2}(0,1)\right) \\
& w(x, T)=0, \quad v(x, T)=u(x, T)
\end{aligned}
$$

The existence of weak solution of the Signorini's problem is obtained by taking the limit when $\varepsilon \rightarrow 0$ of the penalized problem

$$
\begin{aligned}
& a u_{t t}^{\varepsilon}+u_{x x x x}^{\varepsilon}+b \theta_{x x}^{\varepsilon}=f \quad \text { in }(0,1) \times(0, T) \\
& \theta_{t}^{\varepsilon}-\theta_{x x}^{\varepsilon}-c u_{x x t}^{\varepsilon}=g \quad \text { in }(0,1) \times(0, T) \\
& u^{\varepsilon}(x, 0)=u_{0}^{\varepsilon}(x), \quad u_{t}^{\varepsilon}(x, 0)=u_{1}^{\varepsilon}(x), \quad \theta^{\varepsilon}(x, 0)=\theta_{0}^{\varepsilon}(x) \quad \text { in }(0,1) \\
& u^{\varepsilon}(0, t)=u_{x}^{\varepsilon}(0, t)=u_{x x}^{\varepsilon}(1, t)=0 \quad \text { in }(0, T) \\
& \begin{array}{ll}
\theta_{x}^{\varepsilon}(0, t)=\theta^{\varepsilon}(1, t)=0 & \text { in }(0, T)
\end{array} \\
& \sigma^{\varepsilon}(1, t)=-\frac{1}{\varepsilon}\left\{\left[u^{\varepsilon}(1, t)-g_{2}\right]^{+}\right. \\
& \left.-\left[g_{1}-u^{\varepsilon}(1, t)\right]^{+}\right\}-\varepsilon u_{t}^{\varepsilon}(1, t) \quad \text { in }(0, T) \text {. }
\end{aligned}
$$


The term $-\varepsilon u_{t}^{\varepsilon}(1, t)$ was introduced to get the uniqueness of the solution of the problem 13. In that follows, we omit the index $\varepsilon$. The weak formulation of the penalized problem is given by

$$
\begin{aligned}
& u \in W^{1, \infty}\left(0, T ; L^{2}(0,1)\right) \cap L^{\infty}(0, T ; K) \\
& \theta \in L^{2}(0, T ; E) \cap L^{\infty}\left(0, T ; L^{2}(0,1)\right)
\end{aligned}
$$

with

$$
u_{x x t} \in L^{\infty}\left(0, T ; L^{2}(0,1)\right) \text { and } \theta_{x t} \in L^{2}\left(0, T ; L^{2}(0,1)\right)
$$

such that

$$
\begin{aligned}
& -a \int_{0}^{T} \int_{0}^{1} u_{t} v_{t} d x d t+\int_{0}^{T} \int_{0}^{1} u_{x x} v_{x x} d x d t-b \int_{0}^{T} \int_{0}^{1} \theta_{x} v_{x} d x d t \\
& =\int_{0}^{T} \int_{0}^{1} f v d x d t+a \int_{0}^{1} u_{1} v(x, 0) d x \\
& \quad-\frac{1}{\varepsilon} \int_{0}^{T}\left\{\left[u(1, t)-g_{2}\right]^{+}-\left[g_{1}-u(1, t)\right]^{+}\right\} v(1, t) d t-\varepsilon \int_{0}^{T} u_{t}(1, t) v(1, t) d t \\
& -\int_{0}^{T} \int_{0}^{1} \theta w_{t} d x d t+\int_{0}^{T} \int_{0}^{1} \theta_{x} w_{x} d x d t+c \int_{0}^{T} \int_{0}^{1} u_{x x} w_{t} d x d t \\
& =\int_{0}^{1} \theta_{0} w(x, 0) d x-c \int_{0}^{1} u_{0 x x} w(x, 0) d x+\int_{0}^{T} \int_{0}^{1} g w d x d t \\
& \forall v \in L^{2}(0, T ; V), \forall w \in L^{2}(0, T ; E) \text { and } \forall w_{t} \in L^{2}\left(0, T ; L^{2}(0,1)\right), \\
& \text { with } v(x, T)=w(x, T)=0
\end{aligned}
$$
theorem.

We summarize the well posedness of the penalized problem in the following

Theorem 3.2. Let us take

$u_{0} \in K \cap H^{4}(0,1), \quad u_{1} \in H^{3}(0,1), \quad \theta_{0} \in E \cap H^{2}(0,1) \quad$ and $f, g \in H^{1}\left(0, T ; L^{2}(0,1)\right)$

Then, for any $\epsilon>0$, there exists only one strong solution to problem (14)-(15) with the regularity

$$
\begin{aligned}
& u \in W^{2, \infty}\left(0, T ; L^{2}(0,1)\right) \cap W^{1, \infty}(0, T ; V) \cap L^{\infty}\left(0, T ; H^{4}(0,1) \cap V\right) \\
& \theta \in L^{\infty}\left(0, T ; H^{2}(0,1) \cap E\right) \cap W^{1, \infty}\left(0, T ; L^{2}(0,1)\right)
\end{aligned}
$$

Proof. The proof is based on the Galerkin method. Following the same procedures as in [12], we are able to show the existence and uniqueness of solution as indicated in the above Theorem. So, we omit here the proof. On the other hand, using the same arguments as in Kuttler and Shillor in [6], we can show that there exists a 
subsequence of $\left(u^{\varepsilon}, u_{t}^{\varepsilon}, \theta^{\varepsilon}\right)$, we still denote in the same way, that converges to the Signorini's problem; that is

$$
\left(u^{\varepsilon}, u_{t}^{\varepsilon}, \theta^{\varepsilon}\right) \stackrel{*}{\rightarrow}\left(u, u_{t}, \theta\right) \text { in } L^{\infty}(0, T ; V) \times L^{\infty}\left(0, T ; L^{2}(0,1)\right) \times L^{\infty}\left(0, T ; L^{2}(0,1)\right)
$$

when $\left(u, u_{t}, \theta\right)$ satisfies Definition 3.1.

So we have:

Theorem 3.3. Let us $u_{0} \in V, u_{1}, \theta_{0} \in L^{2}(0,1)$ and $f, g \in H^{1}\left(0, T ; L^{2}(0,1)\right)$. Then, there exists a weak solution for Signorini's problem (4)-(11).

Remark 3.4. The uniqueness of the solution to Signorini's problem (4)-(11) remains an open question, as well as the existence result for dimensional space $n \geq 2$.

\section{EXPONENTIAL DECAY}

Next, we show that the solution of the Signorini's problem decays exponentially to zero as time goes to infinity. To show this, we introduce the energy functional associated with the thermoelastic system

$$
E(t ; u, \theta)=E(t)=\frac{1}{2} \int_{0}^{1}\left[a\left|u_{t}\right|^{2}+\left|u_{x x}\right|^{2}+\frac{b}{c}|\theta|^{2}\right] d x
$$

We denote $E_{\varepsilon}(t)=E\left(t ; u^{\varepsilon}, \theta^{\varepsilon}\right)$. The energy of the penalized problem is defined as

$$
\mathscr{E}(t)=E_{\varepsilon}(t)+\frac{1}{\varepsilon} \mathcal{N}_{\varepsilon}(t)
$$

where the functional $\mathcal{N}_{\varepsilon}(t)$ is given by

$$
\mathcal{N}_{\varepsilon}(t)=\frac{1}{2}\left|\left[u^{\varepsilon}(1, t)-g_{2}\right]^{+}\right|^{2}+\frac{1}{2}\left|\left[g_{1}-u^{\varepsilon}(1, t)\right]^{+}\right|^{2}
$$

First, we show that the energy associated with the penalized problem $\mathscr{E}(t)$ decays exponentially to zero as time goes to infinity with rates that do not depend on $\varepsilon$, and later we will pass to the limit when $\varepsilon \rightarrow 0$ to obtain the decay of the energy $E(t)$ associated with the Signorini's problem. The idea is to construct a Lyapunov's functional $\mathscr{L}$, satisfying

$$
\beta_{1} \mathscr{E}(t) \leq \mathscr{L}(t) \leq \beta_{2} \mathscr{E}(t) \text { and } \frac{d}{d t} \mathscr{L}(t) \leq-\beta \mathscr{L}(t), \quad \forall t>0
$$

where $\beta_{1}, \beta_{2}$ and $\beta$ are positive constants. 
Let us consider the penalized problem (13). For simplicity, we take $f=g=0$ in (14) and (15) and we omit the index $\varepsilon$. Using (14) and (15), with $v=u_{t}$ and $w=\theta$, respectively, we arrive at

$$
\begin{aligned}
\frac{1}{2} \frac{d}{d t}\{ & a \int_{0}^{1}\left|u_{t}\right|^{2} d x+\int_{0}^{1}\left|u_{x x}\right|^{2} d x+\frac{b}{c} \int_{0}^{1}|\theta|^{2} d x \\
& \left.+\frac{1}{\varepsilon}\left[\left|\left[u(1, t)-g_{2}\right]^{+}\right|^{2}+\left|\left[g_{1}-u(1, t)\right]^{+}\right|^{2}\right]\right\}=-\int_{0}^{1}\left|\theta_{x}\right|^{2} d x-\varepsilon\left|u_{t}(1, t)\right|^{2}
\end{aligned}
$$

From the above identity and (18), we see that

$$
\frac{d}{d t} \mathscr{E}(t) \leq-\int_{0}^{1}\left|\theta_{x}\right|^{2} d x-\varepsilon\left|u_{t}(1, t)\right|^{2}
$$

Let us introduce the functional $\mathscr{f}_{1}$ as

$$
\mathscr{f}_{1}(t)=a \int_{0}^{1} u u_{t} d x
$$

Under the above notations, we have

Lemma 4.1. For any $\delta>0$ small enough, there exists a positive constant $C_{\delta}>0$, such that

$$
\frac{d}{d t} \oiint_{1}(t) \leq a \int_{0}^{1}\left|u_{t}\right|^{2} d x-\frac{2}{\varepsilon} \mathcal{N}(t)-(1-\delta b) \int_{0}^{1}\left|u_{x x}\right|^{2} d x+C_{\delta} \int_{0}^{1}\left|\theta_{x}\right|^{2} d x
$$

Proof. From definition of $f_{1}(t)$, it follows that

$$
\frac{d}{d t} \mathscr{F}_{1}(t)=a \int_{0}^{1}\left|u_{t}\right|^{2} d x+a \int_{0}^{1} u u_{t t} d x
$$

Using the system (13) and integrating by parts, we get

$$
\begin{aligned}
\frac{d}{d t} \mathscr{g}_{1}(t) & =a \int_{0}^{1}\left|u_{t}\right|^{2} d x-\int_{0}^{1} u\left(u_{x x x x}+b \theta_{x x}\right) d x \\
& =a \int_{0}^{1}\left|u_{t}\right|^{2} d x-u(1, t)\left[u_{x x x}(1, t)+b \theta_{x}(1, t)\right]+\int_{0}^{1} u_{x} u_{x x x} d x+b \int_{0}^{1} u_{x} \theta_{x} d x
\end{aligned}
$$

Using (3), (13), inequality $\alpha \beta \leq \delta \alpha^{2}+\delta^{-1} \beta^{2}$, Poincare's inequality (one-dimensional case is $\left(\|u\|_{L^{2}(\Omega)} \leq L\left\|u_{x}\right\|_{L^{2}(\Omega)}\right.$, where $L$ is the Poincare's constant, remembering that in our case $L=1$ ) and integrating by parts, we conclude

$$
\begin{aligned}
\frac{d}{d t} \mathscr{F}_{1}(t) \leq & a \int_{0}^{1}\left|u_{t}\right|^{2} d x+u(1, t) \sigma(1, t)-\int_{0}^{1}\left|u_{x x}\right|^{2} d x+\delta b \int_{0}^{1}\left|u_{x x}\right|^{2} d x+b \delta^{-1} \int_{0}^{1}\left|\theta_{x}\right|^{2} d x \\
\leq & a \int_{0}^{1}\left|u_{t}\right|^{2} d x-\frac{1}{\varepsilon}\left\{\left|\left(u(1, t)-g_{2}\right)^{+}\right|^{2}+\left|\left(g_{1}-u(1, t)\right)^{+}\right|^{2}\right\} \\
& -(1-\delta b) \int_{0}^{1}\left|u_{x x}\right|^{2} d x+C_{\delta} \int_{0}^{1}\left|\theta_{x}\right|^{2} d x .
\end{aligned}
$$


Using (18), our result follows.

Let us introduce the functional

$$
\mathscr{F}_{2}(t)=-a \int_{0}^{1} q u_{t} u_{x} d x
$$

Lemma 4.2. Let us take $q \in C^{3}([0,1])$, then there exists a constant $C_{0}=$ $\max \left\{\frac{3}{2}, b\right\}>0$, such that

$$
\begin{aligned}
\frac{d}{d t} \mathscr{F}_{2}(t) \leq & -q(1) u_{x}(1, t) \sigma(1, t)+\frac{1}{2} q_{x x}(1)\left|u_{x}(1, t)\right|^{2}-\frac{1}{2} \int_{0}^{1} q_{x x x}\left|u_{x}\right|^{2} d x \\
& +C_{0} \int_{0}^{1}\left(1+q_{x}\right)\left|u_{x x}\right|^{2} d x+\frac{1}{2} q(0)\left|u_{x x}(0, t)\right|^{2} \\
& +\frac{b}{2} \int_{0}^{1}\left|q_{x}+q\right|^{2}\left|\theta_{x}\right|^{2} d x-\frac{a}{2} q(1)\left|u_{t}(1, t)\right|^{2}+\frac{a}{2} \int_{0}^{1} q_{x}\left|u_{t}\right|^{2} d x
\end{aligned}
$$

Proof. Note that

$$
\frac{d}{d t} \mathscr{F}_{2}(t)=-a \int_{0}^{1} q u_{t t} u_{x} d x-a \int_{0}^{1} q u_{t} u_{x t} d x
$$

Using the system (13), we obtain

$$
\frac{d}{d t} \mathscr{F}_{2}(t)=\int_{0}^{1} q u_{x}\left(u_{x x x x}+b \theta_{x x}\right) d x-\frac{a}{2} \int_{0}^{1} q \frac{d}{d x}\left|u_{t}\right|^{2} d x
$$

Integrating by parts and using the boundary conditions, it follows that

$$
\begin{aligned}
\frac{d}{d t} \mathscr{F}_{2}(t)= & q(1) u_{x}(1, t)\left[u_{x x x}(1, t)+b \theta_{x}(1, t)\right]-\int_{0}^{1}\left(q_{x} u_{x}+q u_{x x}\right) u_{x x x} d x \\
& -b \int_{0}^{1}\left(q_{x} u_{x}+q u_{x x}\right) \theta_{x} d x-\frac{a}{2} q(1)\left|u_{t}(1, t)\right|^{2}+\frac{a}{2} \int_{0}^{1} q_{x}\left|u_{t}\right|^{2} d x
\end{aligned}
$$

Integrating by parts, yields

$$
\begin{aligned}
& -\int_{0}^{1}\left(q_{x} u_{x}+q u_{x x}\right) u_{x x x} d x \\
& =\int_{0}^{1}\left(q_{x x} u_{x}+q_{x} u_{x x}\right) u_{x x} d x-\frac{1}{2} \int_{0}^{1} q \frac{d}{d x}\left|u_{x x}\right|^{2} d x \\
& =\frac{1}{2} \int_{0}^{1} q_{x x} \frac{d}{d x}\left|u_{x}\right|^{2} d x+\int_{0}^{1} q_{x}\left|u_{x x}\right|^{2} d x+\frac{1}{2} q(0)\left|u_{x x}(0, t)\right|^{2}+\frac{1}{2} \int_{0}^{1} q_{x}\left|u_{x x}\right|^{2} d x \\
& =\frac{1}{2} q_{x x}(1)\left|u_{x}(1, t)\right|^{2}-\frac{1}{2} \int_{0}^{1} q_{x x x}\left|u_{x}\right|^{2} d x+\frac{3}{2} \int_{0}^{1} q_{x}\left|u_{x x}\right|^{2} d x+\frac{1}{2} q(0)\left|u_{x x}(0, t)\right|^{2}
\end{aligned}
$$


Using inequality $\int_{0}^{1} q_{x} u_{x} \theta_{x} d x \leq \frac{1}{2} \int_{0}^{1}\left|q_{x} \theta_{x}\right|^{2} d x+\frac{1}{2} \int_{0}^{1}\left|u_{x}\right|^{2} d x$ and the Poincare's inequality once more, we arrive at

$$
\begin{aligned}
& -b \int_{0}^{1}\left(q_{x} u_{x}+q u_{x x}\right) \theta_{x} d x \\
& \leq \frac{b}{2} \int_{0}^{1}\left|q_{x} \theta_{x}\right|^{2} d x+\frac{b}{2} \int_{0}^{1}\left|u_{x x}\right|^{2} d x+\frac{b}{2} \int_{0}^{1}\left|q \theta_{x}\right|^{2} d x+\frac{b}{2} \int_{0}^{1}\left|u_{x x}\right|^{2} d x \\
& \leq \frac{b}{2} \int_{0}^{1}\left|q_{x}+q\right|^{2}\left|\theta_{x}\right|^{2} d x+b \int_{0}^{1}\left|u_{x x}\right|^{2} d x
\end{aligned}
$$

Substituting (22) and (23) into (21) and using (3), our result follows.

Now let us introduce

$$
\mathscr{F}_{3}(t)=\int_{0}^{1}\left(\int_{0}^{x} \theta(s, t) d s\right) q u_{t} d x .
$$

Lemma 4.3. Let us take $q \in C^{3}([0,1])$. Then, for $\delta>0$ small enough, there exists a constant $C_{\delta}$, such that

$$
\begin{aligned}
\frac{d}{d t} \mathscr{F}_{3}(t) \leq & C_{\delta} \int_{0}^{1}\left[1+q^{2}+(1+q)^{2}+|q(0)|\right]\left|\theta_{x}\right|^{2} d x-\int_{0}^{1}\left(\frac{c q_{x}}{2}-\delta\right)\left|u_{t}\right|^{2} d x \\
& +\frac{c}{2} q(1)\left|u_{t}(1, t)\right|^{2}-\left.\frac{1}{a}\left(\int_{0}^{x} \theta(s, t) d s\right) q(x) \sigma(x, t)\right|_{x=1} \\
& +\frac{\delta}{a}|q(0)|\left|u_{x x}(0, t)\right|^{2}+\frac{2 \delta}{a} \int_{0}^{1}\left|1+q_{x}\right|^{2}\left|u_{x x}\right|^{2} d x \\
& -\frac{3 b}{2 a} \int_{0}^{1} q_{x}|\theta|^{2} d x-\frac{1}{a} \int_{0}^{1}\left(\int_{0}^{x} \theta(s, t) d s\right) q_{x x}\left[u_{x x}+b \theta\right] d x
\end{aligned}
$$

Proof. Integrating $(13)_{2}$ over $(0, x)$ and using the boundary conditions, we get

$$
\int_{0}^{x} \theta_{t}(s, t) d s=\theta_{x}+c u_{x t}
$$

Multiplying the above equation by $q u_{t}$ and integrating over $(0,1)$, we get

$$
\int_{0}^{1}\left(\int_{0}^{x} \theta_{t}(s, t) d s\right) q u_{t} d x=\int_{0}^{1} \theta_{x} q u_{t} d x+c \int_{0}^{1} u_{x t} q u_{t} d x .
$$

From definition of $\mathscr{F}_{3}(t)$, it follows that

$$
\frac{d}{d t} \mathscr{F}_{3}(t)=\int_{0}^{1}\left(\int_{0}^{x} \theta_{t}(s, t) d s\right) q u_{t} d x+\int_{0}^{1}\left(\int_{0}^{x} \theta(s, t) d s\right) q u_{t t} d x
$$


Substituting (13) ${ }_{1}$ and (24) into the above equation, using inequality $\alpha \beta \leq \delta \alpha^{2}+$ $\delta^{-1} \beta^{2}$ and integrating by parts, we see that

$$
\begin{aligned}
\frac{d}{d t} \mathscr{F}_{3}(t)= & \int_{0}^{1} \theta_{x} q u_{t} d x+c \int_{0}^{1} u_{x t} q u_{t} d x-\frac{1}{a} \int_{0}^{1}\left(\int_{0}^{x} \theta(s, t) d s\right) q\left[u_{x x x x}+b \theta_{x x}\right] d x \\
\leq & \delta^{-1} \int_{0}^{1}\left|q \theta_{x}\right|^{2} d x+\delta \int_{0}^{1}\left|u_{t}\right|^{2} d x+\frac{c}{2} q(1)\left|u_{t}(1, t)\right|^{2} d x \\
& -\frac{c}{2} \int_{0}^{1} q_{x}\left|u_{t}\right|^{2} d x-\left.\frac{1}{a}\left(\int_{0}^{x} \theta(s, t) d s\right) q(x) \sigma(x, t)\right|_{x=1} \\
& +\underbrace{\frac{1}{a} \int_{0}^{1}\left[\theta q+\left(\int_{0}^{x} \theta(s, t) d s\right) q_{x}\right] u_{x x x} d x}_{I_{a}} \\
& +\underbrace{\frac{b}{a} \int_{0}^{1}\left[\theta q+\left(\int_{0}^{x} \theta(s, t) d s\right) q_{x}\right] \theta_{x} d x}_{I_{b}}
\end{aligned}
$$

Integrating by parts once more, yields

$$
I_{a}=-\frac{1}{a} q(0) \theta(0, t) u_{x x}(0, t)-\frac{1}{a} \int_{0}^{1}\left[\theta_{x} q+2 \theta q_{x}+\left(\int_{0}^{x} \theta(s, t) d s\right) q_{x x}\right] u_{x x} d x
$$

From Sobolev's imbedding Theorem $\left(\sup |w(x)| \leq\left\|w_{x}\right\|_{L^{2}(0,1)}\right.$, using $\alpha \beta \leq \delta \alpha^{2}+$ $\delta^{-1} \beta^{2}$ and Poincare's inequality, we get

$$
\begin{aligned}
I_{a} \leq & \frac{1}{a}\left|q(0) \| u_{x x}(0, t)\right|\left(\int_{0}^{1}\left|\theta_{x}\right|^{2} d x\right)^{\frac{1}{2}}+\frac{\delta^{-1}}{a} \int_{0}^{1}\left|q \theta_{x}\right|^{2} d x+\frac{\delta}{a} \int_{0}^{1}\left|u_{x x}\right|^{2} d x \\
& +\frac{2 \delta^{-1}}{a} \int_{0}^{1}\left|\theta_{x}\right|^{2} d x+\frac{2 \delta}{a} \int_{0}^{1}\left|q_{x} u_{x x}\right|^{2} d x-\frac{1}{a} \int_{0}^{1}\left(\int_{0}^{x} \theta(s, t) d s\right) q_{x x} u_{x x} d x \\
\leq & \frac{\delta}{a}\left|q(0) \| u_{x x}(0, t)\right|^{2}+C_{\delta} \int_{0}^{1}\left|\theta_{x}\right|^{2} d x+C_{\delta} \int_{0}^{1}|1+q|^{2}\left|\theta_{x}\right|^{2} d x \\
& +\frac{2 \delta}{a} \int_{0}^{1}\left|1+q_{x}\right|^{2}\left|u_{x x}\right|^{2} d x-\frac{1}{a} \int_{0}^{1}\left(\int_{0}^{x} \theta(s, t) d s\right) q_{x x} u_{x x} d x
\end{aligned}
$$

Integrating by parts, we obtain

$$
I_{b} \leq-\frac{b}{2 a} q(0)|\theta(0, t)|^{2}-\frac{3 b}{2 a} \int_{0}^{1} q_{x}|\theta|^{2} d x-\frac{b}{a} \int_{0}^{1}\left(\int_{0}^{x} \theta(s, t) d s\right) q_{x x} \theta d x
$$

By Sobolev's imbedding, we get

$$
I_{b} \leq C_{\delta}|q(0)| \int_{0}^{1}\left|\theta_{x}\right|^{2} d x-\frac{3 b}{2 a} \int_{0}^{1} q_{x}|\theta|^{2} d x-\frac{b}{a} \int_{0}^{1}\left(\int_{0}^{x} \theta(s, t) d s\right) q_{x x} \theta d x
$$

Substituting $\left(I_{a}\right)$ and $\left(I_{b}\right)$ into (25), Lemma follows. 
To estimate the boundary terms in Lemmas 4.2 and 4.3, we take $q(x)=x-1$. Thus, we have that there exists constant $C_{0}>0$ and for $\delta>0$ small enough, there exists $C_{\delta}>0$, such that

$$
\begin{aligned}
\frac{d}{d t} \mathscr{F}_{2}(t) \leq & C_{0} \int_{0}^{1}\left|u_{x x}\right|^{2} d x-\frac{1}{2}\left|u_{x x}(0, t)\right|^{2}+C_{0} \int_{0}^{1}\left|\theta_{x}\right|^{2} d x+\frac{a}{2} \int_{0}^{1}\left|u_{t}\right|^{2} d x \\
\frac{d}{d t} \mathscr{F}_{3}(t) \leq & C_{\delta} \int_{0}^{1}\left|\theta_{x}\right|^{2} d x-\left(\frac{c}{2}-\delta\right) \int_{0}^{1}\left|u_{t}\right|^{2} d x+\frac{\delta}{a}\left|u_{x x}(0, t)\right|^{2} \\
& +\frac{8 \delta}{a} \int_{0}^{1}\left|u_{x x}\right|^{2} d x-\frac{3 b}{2 a} \int_{0}^{1}|\theta|^{2} d x
\end{aligned}
$$

We also need the following result.

Lemma 4.4. For any $q \in C^{3}([0,1])$, the functionals $\mathscr{F}_{1}, \mathscr{F}_{2}$ and $\mathscr{F}_{3}$ defined in Lemmas 4.1, 4.2 and 4.3, respectively, satisfy

$$
\left|\mathscr{g}_{i}(t)\right| \leq k_{i} E(t), \text { where } i=1,2,3
$$

Proof. From the definition of $\mathscr{f}_{1}(t)$ and by using $\alpha \beta \leq \frac{1}{2} \alpha^{2}+\frac{1}{2} \beta^{2}$ and the Poincare's inequality, we obtain

$$
\left.\left|\mathscr{F}_{1}(t)\right|=\left|a \int_{0}^{1} u u_{t} d x\right| \leq \frac{a}{2} \int_{0}^{1}\left|u_{t}\right|^{2} d x+\frac{a}{2} \int_{0}^{1}\left|u_{x x}\right|^{2} d x \leq k_{1} E(t)\right)
$$

With similar procedure, we can show the same estimates for $\mathscr{F}_{2}(t)$ and $\mathscr{f}_{3}(t)$.

Now, we are able to show exponential stability of Signorini's problem.

Theorem 4.5. The solution of problem (4)-(11) decays exponentially to zero as time goes to infinity; that is, there exist constants $\beta_{0}, \beta>0$, such that the energy of system (4)-(11), satisfies

$$
E(t) \leq \beta_{0} E(0) e^{-\beta t} \quad \forall t \geq 0
$$

Proof. Denote by

$$
\mathscr{F}(t)=\frac{c}{16} \mathscr{F}_{1}(t)+\delta \mathscr{F}_{2}(t)+\frac{a}{4} \mathscr{F}_{3}(t)
$$

where $\delta$ is the constant chosen as in Lemmas 4.1 and 4.3. Consider the Lyapunov's functional defined by

$$
\mathscr{L}(t)=N \mathscr{E}(t)+\mathscr{F}(t)
$$

where $N>0$ is constant. Then, from (19), (20), (26) and (27), it follows

$$
\frac{d}{d t} \mathscr{L}(t) \leq-\left[N-\left(\frac{c C_{\delta}}{16}+\delta C_{0}+\frac{a C_{\delta}}{4}\right)\right] \int_{0}^{1}\left|\theta_{x}\right|^{2} d x
$$




$$
\begin{aligned}
& -\left[\frac{c}{16}-\delta\left(\frac{b c}{16}+C_{0}+2\right)\right] \int_{0}^{1}\left|u_{x x}\right|^{2} d x-\frac{\delta}{4}\left|u_{x x}(0, t)\right|^{2}-\frac{c}{8 \varepsilon} \mathcal{N}(t) \\
& -\left[\frac{a c}{16}-\delta \frac{3 a}{4}\right] \int_{0}^{1}\left|u_{t}\right|^{2} d x-\frac{3 b}{8} \int_{0}^{1}|\theta|^{2} d x-N \varepsilon\left|u_{t}(1, t)\right|^{2}
\end{aligned}
$$

Choosing $N$ large enough and $\delta$ small enough, we conclude that there exist positive constants $a_{i}$, such that

$$
\frac{d}{d t} \mathscr{L}(t) \leq-a_{0} \int_{0}^{1}\left|u_{t}\right|^{2} d x-a_{1} \int_{0}^{1}\left|u_{x x}\right|^{2} d x-a_{2} \int_{0}^{1}|\theta|^{2} d x-\frac{a_{3}}{\varepsilon} \mathcal{N}(t)
$$

Taking $\gamma=\min \left\{a_{0}, a_{1}, a_{2}, a_{3}\right\}$ and using (17), we get

$$
\frac{d}{d t} \mathscr{L}(t) \leq-\gamma \mathscr{E}(t)
$$

From Lemma 4.4 and $N$ large enough, we get that there exist constants $\beta_{1}, \beta_{2}>0$, such that

$$
\beta_{1} \mathscr{E}(t) \leq \mathscr{L}(t) \leq \beta_{2} \mathscr{E}(t)
$$

Combining (28) and (29), we arrive at

$$
\frac{d}{d t} \mathscr{L}(t) \leq-\beta \mathscr{L}(t) \quad \text { where } \beta=\frac{\gamma}{\beta_{2}}
$$

Consequently

$$
\mathscr{E}(t) \leq \beta_{0} \mathscr{E}(0) e^{-\beta t} \quad \forall t \geq 0 \quad \text { where } \beta_{0}=\frac{\beta_{2}}{\beta_{1}}
$$

Here, we obtain the exponential decay of the energy associated with the penalized problem, as time goes to infinity. We will pass to the limit in (30) as $\varepsilon \longrightarrow 0$, to get the decay of the energy associated with the Signorini's problem. From (17), we have

$$
E_{\varepsilon}(t)+\frac{1}{\varepsilon} \mathcal{N}_{\varepsilon}(t) \leq \beta_{0}\left(E_{\varepsilon}(0)+\frac{1}{\varepsilon} \mathcal{N}_{\varepsilon}(0)\right) e^{-\beta t} \quad \forall t \geq 0
$$

Taking the initial data, such that $\mathcal{N}_{\varepsilon}(0)=0$, letting $\varepsilon \longrightarrow 0$, using (16), the lower semicontinuity of the energy and the fact that $\beta$ does not depend on $\varepsilon$, we get that

$$
E(t) \leq \beta_{0} E(0) e^{-\beta t} \quad \forall t \geq 0
$$

Thus, we our conclusion follows. 


\section{NUMERICAL PROBLEM}

To solve the numerical problem, we use the penalized problem (13), assuming enough regularity on the solution. We uncouple the problem (13) as follows (we omit index $\varepsilon$ )

$$
\begin{array}{ll}
a u_{t t}^{n}+u_{x x x x}^{n}+b \theta_{x x}^{n}=0 & \text { in }(0,1) \times(0, T) \\
\theta_{t}^{n}-\theta_{x x}^{n}-c u_{x x t}^{n}=0 & \text { in }(0,1) \times(0, T) \\
u^{n}(x, 0)=u_{0}^{n}(x), \quad u_{t}^{n}(x, 0)=u_{1}^{n}(x), \quad \theta^{n}(x, 0)=\theta_{0}^{n}(x) & \text { in }(0,1) \\
u^{n}(0, t)=u_{x}^{n}(0, t)=u_{x x}^{n}(1, t)=0 & \text { in }(0, T) \\
\theta_{x}^{n}(0, t)=\theta^{n}(1, t)=0 & \text { in }(0, T) \\
\sigma^{n}(1, t)=-\frac{1}{\varepsilon}\left\{\left[u^{n}(1, t)-g_{2}\right]^{+}\right. & \\
\left.\quad-\left[g_{1}-u^{n}(1, t)\right]^{+}\right\}-\varepsilon u_{t}^{n}(1, t) & \text { in }(0, T)
\end{array}
$$

where $n \in \mathbb{N}$. One can show that the solution of the system (31) converge to the solution of system (13), as $n \longrightarrow \infty$. We use finite element method combined with iterative methods to get our solution.

Let us introduce the spaces

$u^{n} \in V=\left\{u \in H^{2}(0,1) ; u(0)=u_{x}(0)=0\right\} \quad$ and $\quad \theta^{n} \in E=\left\{\theta \in H^{1}(0,1) ; \theta(1)=0\right\}$

Then, the variational formulations are given by

$$
\begin{gathered}
a\left(u_{t t}^{n}, v\right)+\left(u_{x x}^{n}, v_{x x}\right)-b\left(\theta_{x}^{n}, v_{x}\right)-\sigma^{n}(1, t) v(1, t)=0 \\
\left(\theta_{t}^{n}, \varphi\right)+\left(\theta_{x}^{n}, \varphi_{x}\right)+c\left(u_{x t}^{n}, \varphi_{x}\right)=0
\end{gathered}
$$

$\forall v \in V_{h}$ and $\forall \varphi \in E_{h}$. With the initial conditions

$$
u^{n}(x, 0)=u_{0}^{n}(x), \quad u_{t}^{n}(x, 0)=u_{1}^{n}(x), \quad \theta^{n}(x, 0)=\theta_{0}^{n}(x)
$$

The approximate problems, semidiscrete and completely discretized by finite element are defined in the usual way (see Hughes [13]). We consider a partition of the interval $[0,1]$ into $P$ subintervals $\left[x_{i-1}, x_{i}\right]$ of length $h$, where $x_{0}=0$ and $x_{P}=1$. Let us denote $\Omega=(0,1)$. For $h \in \mathbb{R}_{+}^{*}$, we consider $V_{h}(\Omega) \subset C^{1}(\Omega)$ and $E_{h}(\Omega) \subset$ $C^{0}(\Omega)$ finite dimensional subspaces given by

$$
V_{h}=\left\{v_{h} \in V ; v_{h}^{e} \in P^{3}\left(\Omega^{e}\right)\right\} \subset V \quad \text { and } \quad E_{h}=\left\{\varphi_{h} \in E ; \varphi_{h}^{e} \in P^{1}\left(\Omega^{e}\right)\right\} \subset E
$$

where $v_{h}^{e}$ and $\varphi_{h}^{e}$ are, respectively, the restrictions of $v_{h}$ and $\varphi_{h}$ to the element " $e$ ", and $P^{1}\left(\Omega^{e}\right)$ and $P^{3}\left(\Omega^{e}\right)$ are, respectively, the sets of linear polynomials and cubical polynomials of Hermite defined in $\Omega^{e}$. For time discretization, we use the Euler implicit method of finite differences, to approximate the terms $u_{t}^{n}, u_{t t}^{n}$ and $\theta_{t}^{n}$. More precisely, the approximations of the terms $u_{t}^{n}, u_{t t}^{n}$ and $\theta_{t}^{n}$ are given by

$$
u_{t}^{n}\left(x, t_{j}\right)=\frac{u_{j}^{n}-u_{j-1}^{n}}{\Delta t}, \quad u_{t t}^{n}\left(x, t_{j}\right)=\frac{u_{j+1}^{n}-2 u_{j}^{n}+u_{j-1}^{n}}{\Delta t^{2}}, \quad \theta_{t}^{n}\left(x, t_{j}\right)=\frac{\theta_{j}^{n}-\theta_{j-1}^{n}}{\Delta t}
$$


The variational problems (32)-(34) completely discretized, consists of finding $u_{h, j}^{n} \in V_{h}$ and $\theta_{h, j}^{n} \in E_{h}$, such that

$$
\begin{aligned}
& \frac{a}{\Delta t^{2}}\left(u_{h, j+1}^{n}, v_{h}\right)+\left(\left(u_{x x}\right)_{h, j+1}^{n},\left(v_{x x}\right)_{h}\right) \\
& \quad=\frac{a}{\Delta t^{2}}\left(2 u_{h, j}^{n}-u_{h, j-1}^{n}, v_{h}\right)+b\left(\left(\theta_{x}\right)_{h, j}^{n},\left(v_{x}\right)_{h}\right)+\sigma_{P, j}^{n} v_{P, j} \\
& \frac{1}{\Delta t}\left(\theta_{h, j}^{n}, \varphi_{h}\right)+\left(\left(\theta_{x}\right)_{h, j}^{n},\left(\varphi_{x}\right)_{h}\right) \\
& \quad=\frac{1}{\Delta t}\left(\theta_{h, j-1}^{n}, \varphi_{h}\right)-\frac{c}{\Delta t}\left(\left(u_{x}\right)_{h, j}^{n}-\left(u_{x}\right)_{h, j-1}^{n},\left(\varphi_{x}\right)_{h}\right)
\end{aligned}
$$

$\forall v_{h} \in V_{h}$ and $\forall \varphi_{h} \in E_{h}$. With the initial conditions

$$
u_{h, 0}^{n}(x)=\left(u_{0}\right)_{h}^{n}(x), \quad \frac{u_{h, 1}^{n}(x)-u_{h, 0}^{n}(x)}{\Delta t}=\left(u_{1}\right)_{h}^{n}(x), \quad \theta_{h, 0}^{n}(x)=\left(\theta_{0}\right)_{h}^{n}(x)
$$

Remark 5.1. To get computational results, we use the implemented code in Fortran 90. The graphics were developed using Maple.

To solve the numerical problem, we use the following resolution algorithm:

Given $u_{h, 0}^{n}, u_{h, 1}^{n}, \theta_{h, 0}^{n}$

For $j=1, \ldots, k$

$\theta_{h, j}^{0}=\theta_{h, j-1}, \quad u_{h, j+1}^{0}=u_{h, j}, \quad u_{h, j}^{0}=u_{h, j-1}$

For $n=1, \ldots, N_{p}$, find $\theta_{h, j}^{n} \in E_{h}$ and $u_{h, j+1}^{n} \in V_{h}$, such that

$$
\begin{aligned}
& \quad\left(\theta_{h, j}^{n}, \varphi_{h}\right)+\Delta t\left(\left(\theta_{x}\right)_{h, j}^{n},\left(\varphi_{x}\right)_{h}\right) \\
& \quad=\left(\theta_{h, j-1}^{n}, \varphi_{h}\right)-c\left(\left(u_{x}\right)_{h, j}^{n}-\left(u_{x}\right)_{h, j-1}^{n},\left(\varphi_{x}\right)_{h}\right) \quad \forall \varphi_{h} \in E_{h}, \\
& a\left(u_{h, j+1}^{n}, v_{h}\right)+\Delta t^{2}\left(\left(u_{x x}\right)_{h, j+1}^{n},\left(v_{x x}\right)_{h}\right) \\
& \quad=a\left(2 u_{h, j}^{n}-u_{h, j-1}^{n}, v_{h}\right)+\Delta t^{2} b\left(\left(\theta_{x}\right)_{h, j}^{n},\left(v_{x}\right)_{h}\right)+\Delta t^{2} \sigma_{P, j}^{n} v_{P, j} \quad \forall v_{h} \in V_{h} \\
& \theta_{h, j}=\theta_{h, j}^{N_{p}}, \quad u_{h, j+1}=u_{h, j+1}^{N_{p}}, \quad u_{h, j}=u_{h, j}^{N_{p}}
\end{aligned}
$$

\section{COMPUTATIONAL RESULTS AND CONCLUSION}

The dimensionless constants used in the graphics are given in Table 1 . The penalization parameter is given by $1 / \varepsilon=1 \times 10^{9}$ and we taking the stops in positions $g_{1}=-6 \times 10^{-4}$ and $g_{2}=6 \times 10^{-4}$. We use a uniform mesh with 50 elements and $\Delta t=2 \times 10^{-5}$, and we choose the following initial conditions for $u$ and $\theta$

$$
u(x, 0)=0, \quad \theta(x, 0)=x^{2}-2 x^{3}+x^{4}, \quad u_{t}(x, 0)=-5\left[1-2 x^{2}+\frac{4}{3} x^{3}-\frac{1}{3} x^{4}\right]
$$

We consider the evolution of the beam's solution $u(x, t)$, the beam's oscillations at the end $x=1: u(1, t)$, the energy $E(t)$ and the temperature difference $\theta(x, t)$, in the time $T=0.01$. Thus, we have the graphics: 


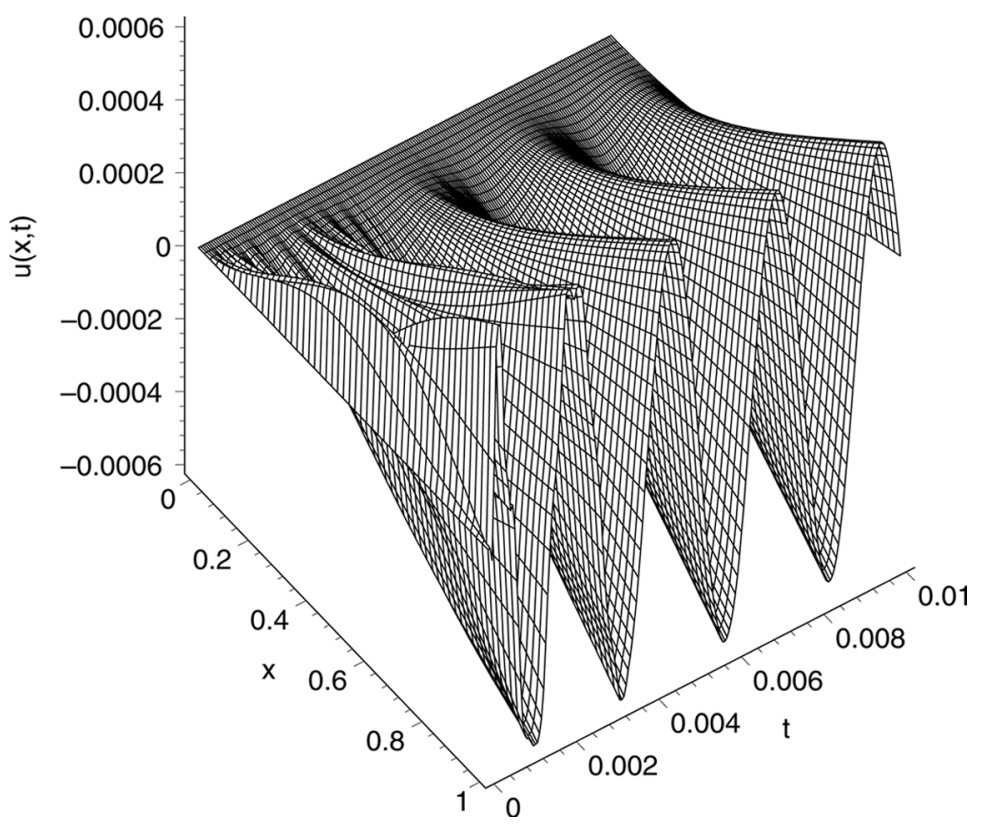

Figure 2 Evolution of the beam's solution: $u(x, t)$.

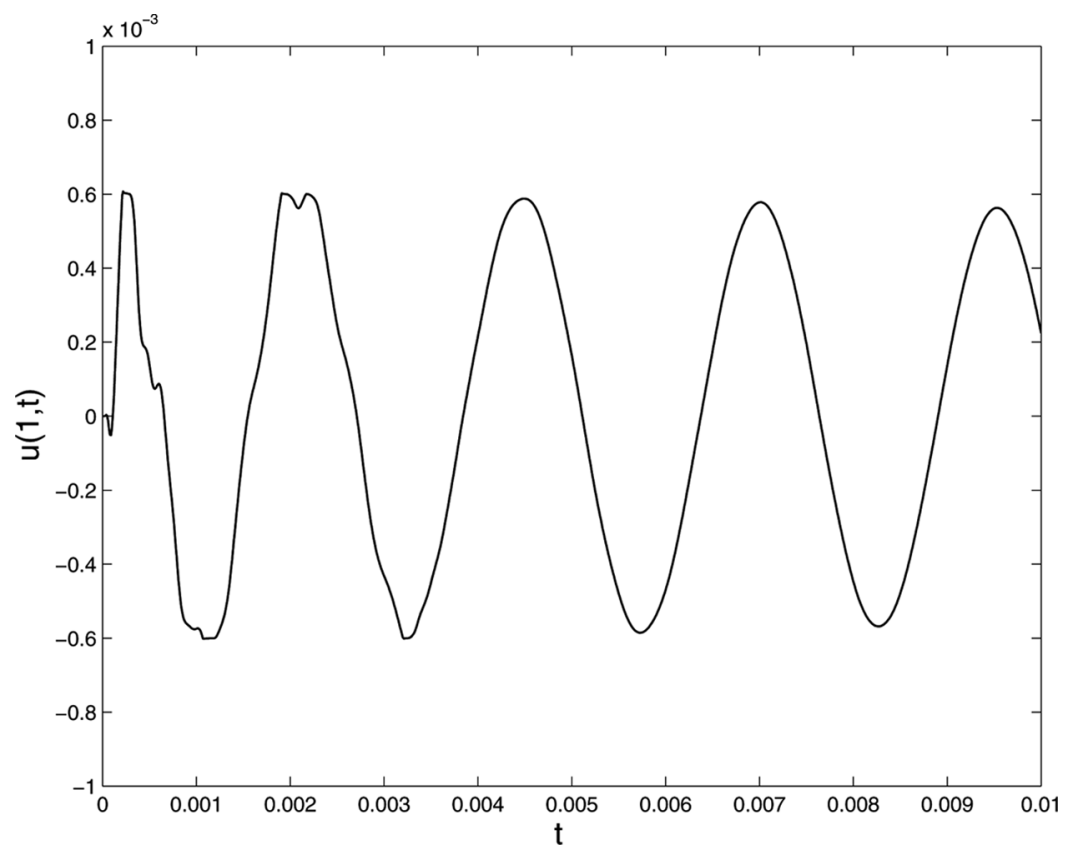

Figure 3 Beam's oscillations at the end $x=1: u(1, t)$. 


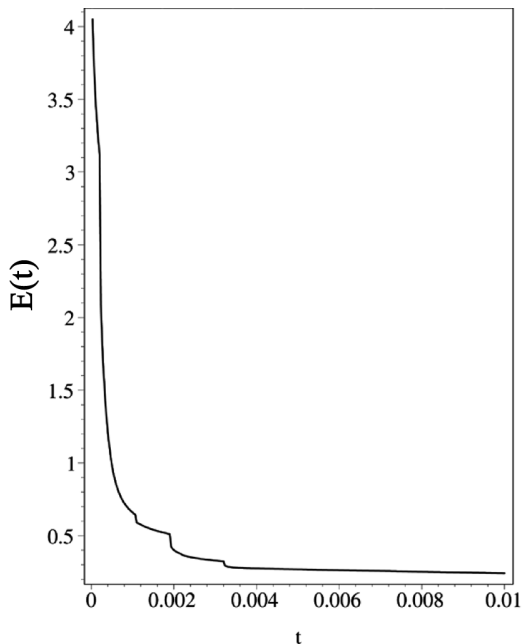

(a)

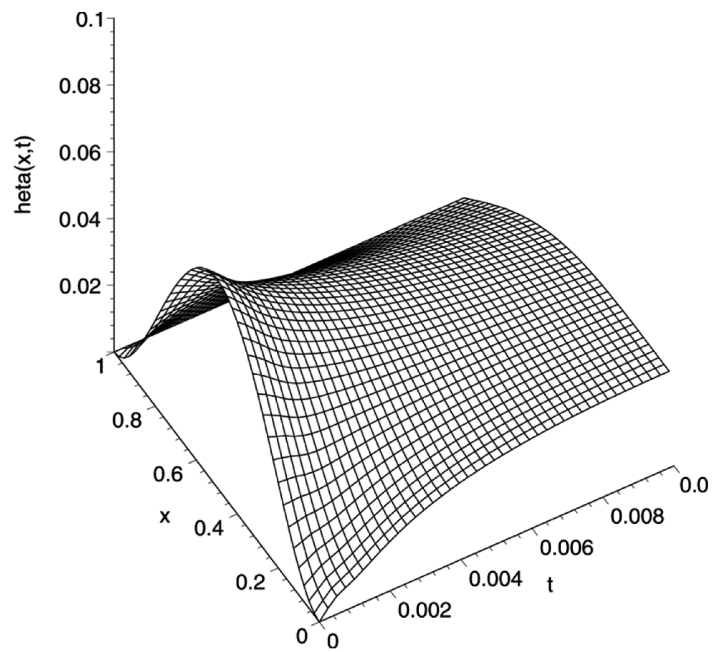

(b)

Figure 4 (a) Energy: $E(t)$. (b) Temperature difference: $\theta(x, t)$.

We observe in Figures 2 and 3 that the trajectory of the displacement $u$ in 1 , remains constrained by the obstacles in the positions $g_{1}$ and $g_{2}$. We notice that initially $u(1, t)$ touches the obstacles and presents irregular behavior due to the inertia effect. However, as time goes by, the solution begins to decay and to regularize itself. In Figure 4(a), we see the decay of the energy that, also, presents some irregularities. In Figure 4(b), we see how the temperature difference is distributed along the beam.

In the next graphics, we consider the beam's oscillations at the end $x=1$ : $u(1, t)$ and the energy $E(t)$, in times $T=0.05$ and $T=0.1$, respectively.

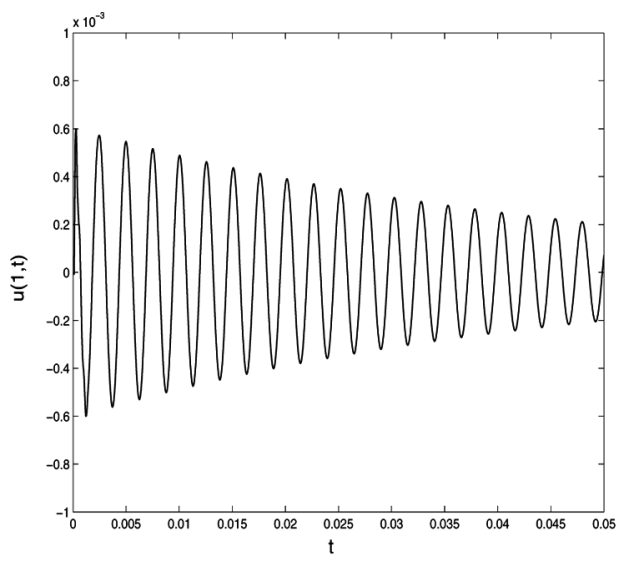

(a)

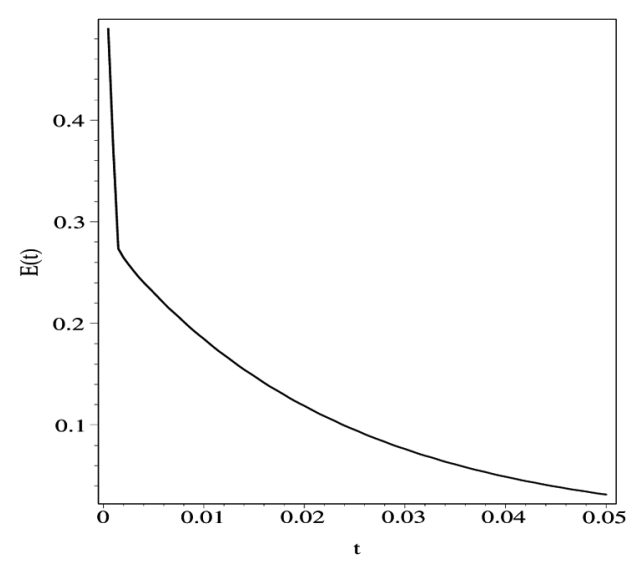

(b)

Figure 5 (a) Beam's oscillations at the end $x=1: u(1, t)$. (b) Energy: $E(t)$. 


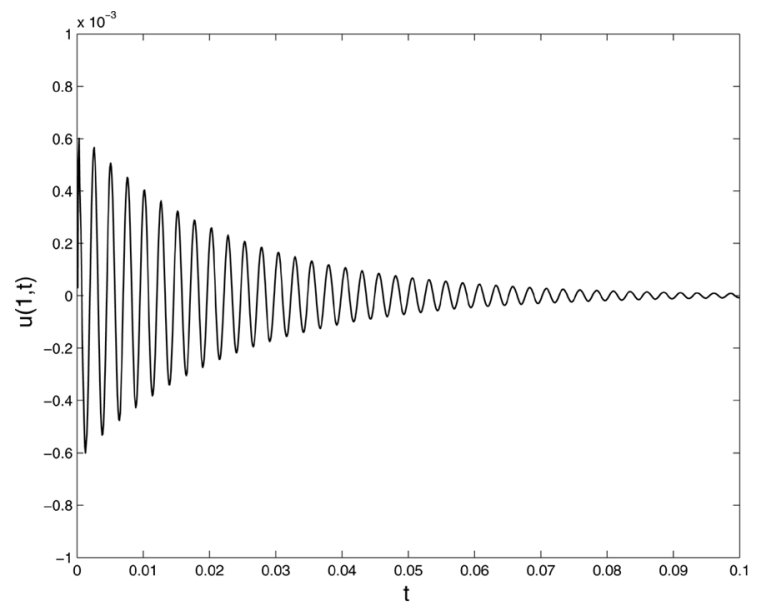

(a)

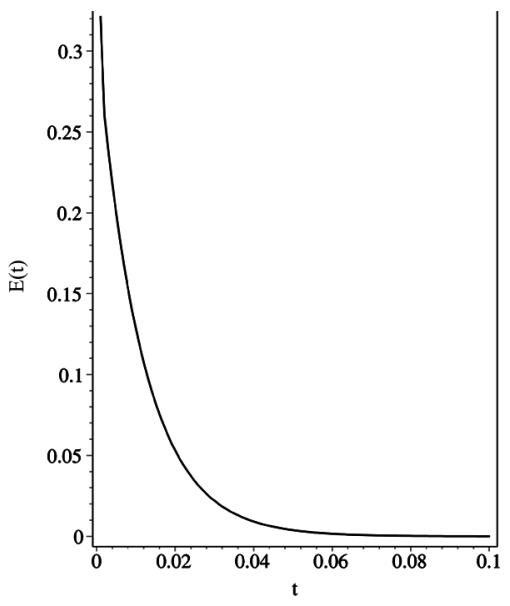

(b)

Figure 6 (a) Beam's oscillations at the end $x=1: u(1, t)$. (b) Energy: $E(t)$.

We notice in Figures 5 and 6 same behavior of the previous graphics. Moreover, as time increases, we see better the exponential decay of the solution.

Finally, we present a case where the penalization parameter is given by $1 / \varepsilon=$ $1 \times 10^{7}$ and analyse the beam's oscillations at the free end $x=1: u(1, t)$, in the time $T=0.05$.

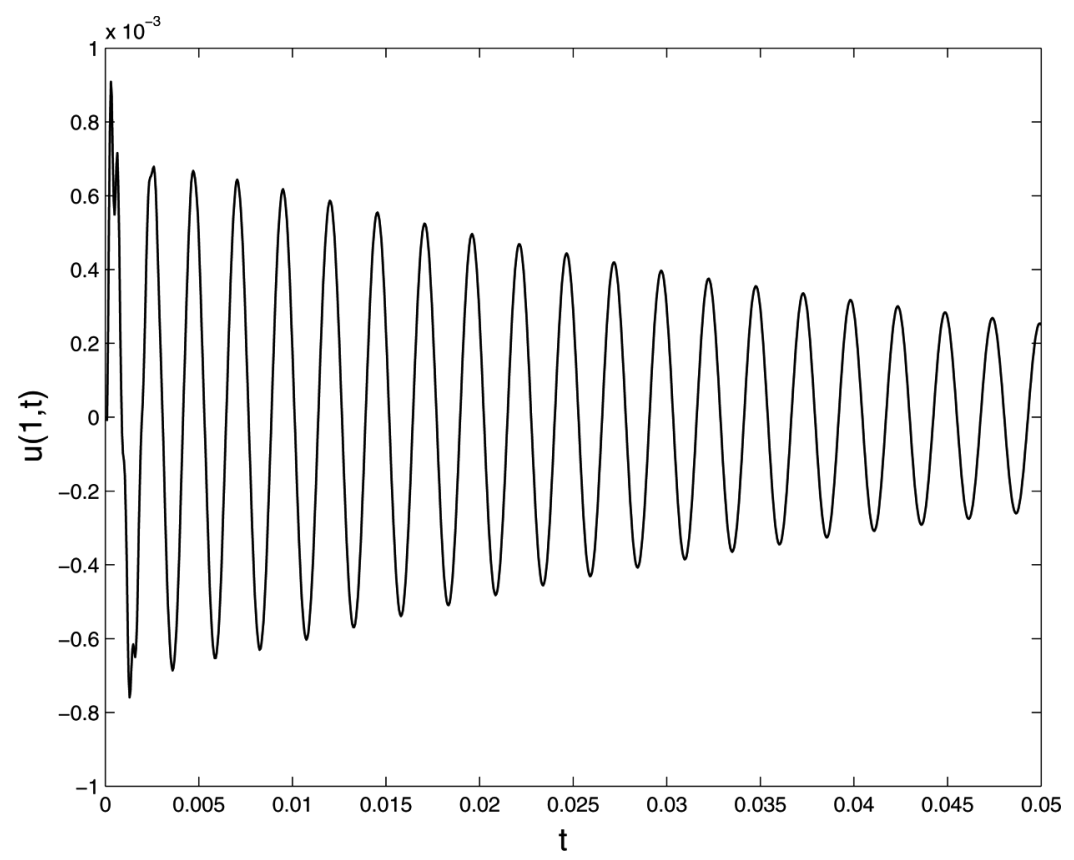

Figure 7 Beam's oscillations at the end $x=1: u(1, t)$. 
In Figure 7 , note that close to zero, these values of $u(1, t)$ in some interval of time are larger than $g_{2}$ and in other interval of time are smaller than $g_{1}$, this is because of the normal compliance condition, which considers the elastic obstacles. As $\varepsilon \longrightarrow 0$, the elastic body goes to a rigid body (see Figure 5(a)); characterizing the numerical convergence of the penalized problem for the Signorini's problem. Through the numerical simulations, we observe that the smaller is the value of $\varepsilon$, the bigger is the computational cost to guarantee the convergence of method.

\section{REFERENCES}

1. K. L. Kuttler, A. Park, M. Shillor, and W. Zhang, Unilateral Dynamic Contact of Two Beams, Mathematical and Computer Modelling, vol. 34, pp. 365-384, 2001.

2. K. T. Andrews, M. Shillor, and S. Wright, On the Dynamic Vibrations of an Elastic Beam in Frictional Contact with a Rigid Obstacle, J. Elast., vol. 42, pp. 1-30, 1996.

3. Y. Dumont, K. L. Kuttler, and M. Shillor, Analysis and Simulations of Vibrations of a Beam with a Slider, J. Eng. Math., vol. 47, pp. 61-82, 2003.

4. J. Bajkowski, J. R. Fernández, K. L. Kuttler, and M. Shillor, A Thermoviscoelastic Beam Model for Brakes, Euro. J. Appl. Math., vol. 15, pp. 181-202, 2004.

5. F. C. Moon and S. W. Shaw, Chaotic Vibrations of a Beam with Nonlinear Boundary Conditions, J. Non-Linear Mech., vol. 18, pp. 465-477, 1983.

6. K. L. Kuttler and M. Shillor, Vibrations of a Beam Between Two Stops. Dynamics of Continuous, Discrete Impulsive Sys. Ser. B: Appl.-Algorithms, vol. 8, pp. 93-110, 2001.

7. Y. Dumont and L. Paoli, Vibrations of a Beam Between Stops: Convergence of a Fully Discretized Approximation, Math. Model. Numer. Anal., vol. 40, pp. 705-734, 2006.

8. Y. Dumont, Vibrations of a Beam Between Stops: Numerical Simulations and Comparison of Several Numerical Schemes, Math. Comput. Simul., vol. 60(1-2), pp. 45-83, 2002.

9. A. N. Norris, Dynamics of Thermoelastic Thin Plates: A Comparison of Four Theories, Journal of Thermal Stresses, vol. 29, pp. 169-195, 2006.

10. J. E. Lagnese and J. L. Lions, Modelling Analysis and Control of Thin Plates, Collection RMA, p. 175, Masson, Paris, 1989.

11. M. I. M. Copetti, Finite Element Approximation to a Contact Problem in Linear Thermoelasticity, Math. Comput., vol. 68, pp. 1013-1024, 1999.

12. J. E. M. Rivera and S. Jiang, The Thermoelastic and Viscoelastic Contact of Two Rods, J. Math. Anal. Appl., vol. 217, pp. 423-458, 1998.

13. T. J. R. Hughes, The Finite Element Method: Linear Static and Dynamic Finite Element Analysis, p. 682, Dover Publications, Mineola, New York, 2000.

14. J. R. Barber and R. Zhang, Transient Behaviour and Stability for the Thermoelastic Contact of two Rods of Dissimilar Materials, Int. J. Mech. Sci., vol. 30, pp. 691-704, 1988 . 